\title{
Automatisierte Leistungsabwicklung in der Krankenversicherung
}

Urs Zellweger

Dipl. Informatik-Ingenieur ETHZ, Helsana Versicherungen AG, Mitglied der Direktion
Korrespondenz: Urs Zellweger

Helsana Versicherungen AG Postfach

CH-8081 Zürich

urs.zellweger@helsana.ch
Die Leistungsabwicklung in der Krankenversicherung (KV) hat zum Ziel, erbrachte Leistungen auf deren Wirksamkeit, Zweckmässigkeit und Wirtschaftlichkeit zu prüfen und sie zu vergüten. Für die Anlieferung der zu prüfenden Rechnungen spielt der elektronische Datenaustausch (eDA) eine entscheidende Rolle. Es können erhebliche Kosteneinsparungen bei gleichzeitiger Qualitätssteigerung für Leistungserbringer und Kostenträger erreicht werden. Durch die Einführung von TARMED 2004 wurden die Vertragspartner auf eine einheitliche Datenstruktur und zur elektronischen Abrechnung verpflichtet. Die Rechnungsvolumina haben dadurch im eDA massiv zugenommen. Der einheitliche Standard wird zunehmend von weiteren Leistungserbringern für die Rechnungsübermittlung genutzt. Zusätzlich zu den Rechnungen müssen aber auch Leistungsermächtigungen (Kostengutsprachen, Überweisungen, Verordnungen und Aufträge) verarbeitet werden. Diese können heute jedoch noch nicht elektronisch übermittelt werden.

\section{Manuel sur les transformations du système de santé suisse}

Le système de santé suisse est en profonde mutation. Les moteurs principaux à la base de ces transformations sont la concurrence croissante entre acteurs, les nouveaux modèles de financement et de conventions entre ceux qui fournissent des prestations et ceux qui les financent, de même que l'évolution démographique et épidémiologique observée dans notre société. Les adaptations apportées aux conditions cadres légales augmentent la pression sur les acteurs. Les hôpitaux, tous les fournisseurs de prestations, les assureurs-maladie et l'industrie devront, dans les 2 à 5 ans, orienter leurs stratégies et les processus organisationnels sur ces facteurs de changement.

Ces thèses forment la trame du livre qui vient de paraître, uniquement en allemand, sous le titre «Gesundheitswesen Schweiz im Umbruch» et dans lequel 40 experts de la recherche, des sciences et de la pratique examinent attentivement, au fil de 50 articles spécialisés, les caractéristiques du système de santé
Rechnungen elektronisch auszutauschen ist in den Systemen des «tiers garant» (TG) und «tiers payant» (TP) möglich. Da jedoch eine TG-Rechnung immer vom Versicherten in Papierform eingereicht wird, ist der Aufwand bei einer elektronischen TP-Rechnung erheblich geringer als bei einer elektronischen TG-Rechnung. Die Erlaubnis, die Rechnungsdaten beim Leistungserbringer elektronisch beziehen zu dürfen, wird der KV nur durch das Einreichen der Papierrechnung erteilt. Auch diese Rechnung muss also gescannt und digitalisiert werden und kann erst dann mit den elektronischen Detaildaten angereichert werden.

2006 haben die 87 KV in der Grundversicherung Bruttoleistungen von 20,6 Mia. Franken für 7,5 Mio. Personen ausbezahlt. Diese Zahlen haben folgende Basis: 55 Mio. Rechnungen und 20 Mio. Leistungsermächtigungen (davon über die Hälfte Medikamentenrezepte) sind von über 50000 Leistungserbringern (davon etwa 30000 Leistungserbringer nach KVG) verarbeitet wor-

suisse tout en exposant en détail les modifications qui se produiront au cours des prochaines années. On y relève les questions centrales suivantes: qu'est-ce qui rendra le système de santé suisse plus complexe à l'avenir? Quelles seront les répercussions de cette complexité accrue et quels processus deviendront-ils vitaux? Quelles mesures permettront-elles de développer la vitalité aux fins d'anticiper l'augmentation de la complexité?

Les éditeurs de cet ouvrage, à savoir Willy Oggier, Andreas Walter, Serge Reichlin et Michael Egli, sont quatre personnalités disposant d'une expérience professionnelle de longue date dans les domaines les plus divers de la santé publique. Quelques auteurs ont rédigé pour le BMS un article sur un thème central du manuel. Nous présentons leurs réflexions à notre lectorat dans les numéros 15 à 18-19/2008 sous forme de série brève.

Vous trouverez de plus amples informations sur le manuel «Gesundheitswesen Schweiz im Umbruch» sous www.ehealthcare.ch 
den. Vor der Auszahlung musste die korrekte Anwendung von rund 100 Tarifen mit total über 130000 Positionen geprüft werden.

\section{Anforderungskriterien an die Leistungsabwicklung}

Ein Leistungsabwicklungssystem in der KV muss zwingend folgenden Anforderungen genügen:

- Rechnungen und Leistungsermächtigungen müssen verarbeitet werden können. Allein die Helsana-Gruppe verarbeitete 2006 jede Stunde 5000 Rechnungen mit durchschnittlich 10 Einzelpositionen und zahlte pro Stunde rund 2 Mio. Franken aus. Die meisten eingereichten Rechnungen sind strukturiert und standardisiert. Es gibt aber auch Rechnungen, welche vollkommen unstrukturiert auf Quittungszetteln aus dem Supermarkt eingereicht werden.

- Abspeicherung von mehreren Millionen Einzelleistungen, die (bei Helsana) für über eine Million Versicherte von Zehntausenden verschiedenen Leistungserbringern erbracht werden.

- Verwaltung und Prüfung von mehreren tausend Vertrags- und Gesetzesregeln entsprechend ihrer Gültigkeit, da sie sich über die Zeit ändern.

- Für jeden einzelnen Beleg aus den Tausenden von Regeln die relevanten identifizieren und bis zu 5000 Einzelprüfungen durchführen.

\section{Praxisbeispiel}

Was bedeuten diese Anforderungen konkret? Zur Illustration ein Praxisbeispiel: Die schwangere Hausfrau Kristina Muster (versichert bei SANASwiss) stolpert auf dem Weg zur routinemässigen Ultraschalluntersuchung und fällt auf ihr Knie. Dr. Frauenknecht verarztet vor der Ultraschalluntersuchung das verletzte Knie. Zusätzlich nimmt er noch Blut, das er dem Labor Hämaglobi zustellt. Frau Muster stellt er ein Rezept für den Bezug der schmerzstillenden entzündungshemmenden Salbe Antiweh und ein Medikament zur Linderung der Schwangerschaftsbeschwerden aus.

Aus diesem Beispiel ergeben sich drei Rechnungen:

1. Der Gynäkologe Frauenknecht stellt seine Leistungen zusammen mit den vorangehenden und der kommenden Konsultation in Rechnung und stellt diese Frau Muster zur Zahlung zu. Diese sendet die Rechnung erst nach der Geburt an SANASwiss.

2. Das Labor Hämaglobi stellt im Verlauf der kommenden Woche die Rechnung der Versicherung SANASwiss zu.
3. Frau Muster bezieht die Medikamente bei der Apotheke, die gegen das Rezept die Originalsalbe Antiweh abgibt, obwohl das Generikum Ge2Antiweh existiert.

Selbst bei diesem einfachen Beispiel werden die meisten Versicherungen Mühe haben, die Rechnung korrekt nach Gesetz abzurechnen. Bei der KV SANASwiss ergibt sich nun folgender chronologischer Eingang der Belege mit den zugehörigen Prüfungen:

1. Die Apothekenrechnung wird von der Zahlstelle elektronisch mit dem Behandlungsgrund Mutterschaft an SANASwiss übermittelt. Die Versicherung prüft die Rechnung auf Korrektheit. Dabei stellt sie dank der grossen Erfahrung der Sachbearbeiterin fest, dass die Salbe Antiweh nichts mit der Schwangerschaft zu tun hat, sondern bei äusseren Verletzungen angewendet wird. Sie muss nun prüfen, ob Frau Muster die Unfallversicherung eingeschlossen hat. Falls ein Unfall vorliegt, müsste es über die Unfallversicherung abgerechnet werden. Da Frau Muster Hausfrau ist, hat sie keine Unfallversicherung durch den Arbeitgeber. Weiter weist das System darauf hin, dass für Antiweh ein Generikum existiert. Die Sachbearbeiterin prüft das Rezept auf einen Vermerk, ob die Patientin eine Unverträglichkeit gegenüber dem Generikum hat. Sie findet aber keinen Hinweis. Daher rechnet sie es mit dem höheren Selbstbehalt von $20 \%$ unter Unfall ab. Die Kostenbeteiligung wird vom Versicherten eingezogen und der volle Betrag der Zahlstelle überwiesen (diese überweist den Betrag an den Apotheker).

2. Die Laborrechnung mit dem Behandlungsgrund Mutterschaft wird elektronisch von Hämaglobi an SANASwiss übermittelt. In der Rechnungsprüfung wird festgestellt, dass das Labor einen Tarif anwendet, der von SANASwiss nicht akzeptiert wird. Die Rechnung wird an Hämaglobi zurückgesandt.

3. Das Labor Hämaglobi stellt die Rechnung erneut mit dem korrekten Tarif zu. SANASwiss stellt keine weiteren Fehler fest und verarbeitet die Rechnung. Die Kostenbeteiligung wird Frau Muster belastet und dem Labor der volle Betrag überwiesen.

4. Frau Muster sendet die Arztrechnung an SANASwiss, zusammen mit der Rechnungskopie, die sie vom Labor erhalten hat.

5. SANASwiss scannt die von Frau Muster eingesandten Rechnungen und verarbeitet sie in ihrem System. Dabei wird festgestellt, dass die Laborrechnung bereits bezahlt wurde. Die Rechnung von Dr. Frauenknecht mit dem 
Behandlungsgrund Mutterschaft wird bearbeitet und vollständig bezahlt. Ein Teil der Behandlung wäre Unfall, wofür Kostenbeteiligung belastet werden müsste. Dies ist jedoch nicht erkennbar. In diesem Fall erhält Frau Muster den gesamten Betrag der Behandlung beim Gynäkologen vergütet, jedoch nichts für die Laborrechnung.

An diesem einfachen Beispiel wird deutlich, welche Abläufe durch einen einzigen Arztbesuch ausgelöst werden. Viele gesetzliche Vorgaben, die auch noch zu prüfen wären (alle Abschnitte der KLV Art. 13, z.B. «gab es nur 2 Ultraschalluntersuchungen, maximal 7 Untersuchungen»), wurden in diesem Beispiel weggelassen.

\section{Ausblick}

Um die Leistungsabwicklung weiter automatisieren zu können, ist von zentraler Bedeutung, dass der elektronische Austausch von Daten weiter gefördert wird. Dazu ist gemeinsam mit den
Leistungserbringern der Gesamtprozess von der Leistungsermächtigung bis zur Rechnung zu analysieren, mit dem Ziel, den Prozess für alle Parteien zu verbessern. Den Grossteil der manuellen Arbeit in der KV machen heute Leistungsermächtigungen aus. Sie und nicht die Verbesserung der Administrativdaten sind der Bereich, der erheblich effizienter auszugestalten ist. Die Versichertenkarte, wie sie in den entsprechenden Verordnungen vorgesehen ist, trägt nichts dazu bei. Sie verursacht für alle Beteiligten nur hohe Kosten.

Der nächste Quantensprung im eDA wird der Austausch von Leistungsermächtigungen sein. Dies wird jedoch nicht ohne einen neuen gemeinsamen Standard möglich sein. Aus diesem Grund unterstützen führende Krankenversicherer einen Standard, der auf einem generellen Modell beruht und die Übermittlung von Rechnungen und Leistungsermächtigungen gleichermassen zulässt. 